Check for updates

Cite this: RSC Adv., 2017, 7, 35537

\title{
Molecular dynamics simulations of nanoscale engravings on an alkanethiol monolayer
}

\author{
Zhengqing Zhang, ${ }^{\mathrm{a}}$ Yoonho $\mathrm{Ahn}{ }^{\mathrm{b}}$ and Joonkyung Jang (D) *a
}

Received 29th May 2017

Accepted 12th July 2017

DOI: $10.1039 / \mathrm{c} 7 \mathrm{ra06005j}$

rsc.li/rsc-advances
Nanoscale patterns engraved on the bulk monolayer of 1-decanethiol were examined using molecular dynamics simulations. The smallest widths required for stable circular and linear engravings were found to be 3.2 and $2.4 \mathrm{~nm}$, respectively. The minimum separations possible without merging for circular and linear trenches were 2.0 and $2.6 \mathrm{~nm}$, respectively. These values set the ultimate the size limit and spatial resolution for engraving the alkanethiol monolayer.

\section{Introduction}

Alkanethiol monolayers with micro- or nano-scale sizes have many applications, including resists for pattern transfers, ${ }^{1,2}$ templates for biomolecules, ${ }^{3-6}$ coatings for controlling surface properties, ${ }^{7-9}$ and circuit components in molecular electronic devices. ${ }^{10,11}$ High-fidelity micro- or nano-patterns of such monolayers can be constructed using a range of techniques, such as micro-contact printing, ${ }^{12,13}$ micro-writing, ${ }^{1,14}$ dip-pen nanolithography, ${ }^{15,16}$ nano-grafting, ${ }^{17}$ and nano-shaving. ${ }^{18,19}$ These patterning methods can be categorized as either a built-up or a dig-down method. The built-up method involves the positive deposition of molecules on a specific region of a substrate, e.g., using an atomic force microscope tip ${ }^{15,16}$ or stamp. ${ }^{12,20}$ Monolayers, tens of nanometers in width, are built up on various substrates. ${ }^{21-23}$ In contrast, dig-down methods, such as nanoshaving, ${ }^{18,19}$ etch out selected regions of a monolayer fully covered with alkanethiol molecules. In this way, circular holes and linear trenches, tens of nms in width, can be engraved. ${ }^{19,24}$

Simulation studies on the self-assembled monolayers of alkanethiols on gold surfaces abound. For example, Rai et al. studied the effects of the flexibility of Au-C-S bond on the structure of an alkanethiol monolayer on a gold (111) surface..$^{25}$ Singh et al. studied the phase separation of a mixed monolayer of alkanethiols with different lengths on a gold nanoparticle. ${ }^{9}$ The wettability of a mixed monolayer of thiols with hydrophobic and hydrophilic terminal groups were simulated by Szöri et al. ${ }^{7}$ and by Vasumathi et al. ${ }^{8}$

Given the ever-decreasing feature sizes of these patterns, the optimal resolution of these patterns is a major research and engineering question. The stability of a thiol monolayer relies on compact and ordered intermolecular packing; alkyl chains are packed together and aligned, tilting slightly ( $\sim 25$ degrees) from the surface normal. The sulfur head groups are also packed together and adsorbed strongly to a surface (typically gold). On the other hand, a finite-sized monolayer will be destabilized as its width decreases to a few nms because of the lower degree of intermolecular packing (of alkyl chains and sulfur groups). These finite-sized monolayers also have new structural features, such as periodic changes in the tilt orientation of molecules. ${ }^{26,27}$ Previously, the nanoscale monolayers of octadecanethiol (ODT) grown via a built-up method were studied using molecular dynamics (MD) simulations. ${ }^{23,26}$ The stability and structure of the nanoscale monolayers of ODT were examined by varying the widths of these monolayers to a few nms.

The ultimate size limit and spatial resolution of a nanoscale engraving on a thiol monolayer are unknown, even though these monolayers are used widely in many applications. ${ }^{5,28,29}$ Various patterns engraved on the monolayers of alkanethiol are utilized as resists for pattern transfers, ${ }^{1,2}$ the templates for biomolecules, ${ }^{3-6}$ and the circuit components in molecular electronic devices. ${ }^{10,11}$ Given the feature sizes of these negative patterns become smaller and smaller, a fundamental design principle for fabricating such patterns is desired. The present study examined circular and linear engravings on a monolayer of 1-decanethiol (DT), $\mathrm{CH}_{3}\left(\mathrm{CH}_{2}\right)_{8} \mathrm{CH}_{2} \mathrm{SH}$, using MD simulation. DT is a prototypical molecule previously used for controlling surface wettabilities, ${ }^{30,31}$ for molecular devices, ${ }^{32}$ for sensors,${ }^{33,34}$ and for protective coatings. ${ }^{35}$ By analyzing the molecular packing structures and thermal stabilities of various engraved patterns, the present simulation revealed the size limits of stable circular and linear patterns engraved on a DT monolayer. How close two engraved patterns can be without coalescing into one was also studied to provide a theoretical limit to the spatial resolution of an engraved pattern on a DT monolayer.

\section{Simulation methods}

${ }^{a}$ Department of Nanoenergy Engineering, Pusan National University, Busan 609-735, South Korea.E-mail:jkjang@pusan.ac.kr

${ }^{b}$ Department of Applied Physics, Kyung Hee University, Yongin 446-701, South Korea
The $\mathrm{CH}_{3}, \mathrm{CH}_{2}$ and $\mathrm{SH}$ groups of DT were treated as united atoms ${ }^{36,37}$ to save simulation time without losing the accuracy of 
an all-atom simulation. ${ }^{38,39}$ The tilt direction of each molecule was calculated by selecting the $\mathrm{C}_{2}, \mathrm{C}_{4}$, and $\mathrm{C}_{6}$ atoms of DT. Three methyl groups at the tail $\left(\mathrm{C}_{8}, \mathrm{C}_{9}\right.$, and $\left.\mathrm{C}_{10}\right)$ were excluded because they contain many gauche defects. The tilt direction vector of the $i^{\text {th }}$ molecule, $\vec{u}_{i}$, was defined as the average of the vectors from the sulfur atom to these carbon atoms (Fig. 1). ${ }^{36,40}$ The tilt angle of the $i^{\text {th }}$ molecule, $\theta_{i}$, was given by the polar angle of $\vec{u}_{i}$ measured from the surface normal. The surface projection of $\vec{u}_{i}$ was designated by $\vec{v}_{i}$. The order parameter of the molecular orientation, $O_{\mathrm{u}}$, was defined as ${ }^{41}$

$$
O_{\mathrm{u}}=\left\langle 0.5\left[\left(\vec{u}_{i} \cdot \vec{u}_{j}\right)^{2}-1\right]\right\rangle_{i \neq j},
$$

where \langle\rangle$_{i \neq j}$ represents the average over all the intermolecular pairs.

The bond stretching and bending angle interactions were modeled using the harmonic potentials. ${ }^{42}$ The four-atom torsion potential $(\mathrm{C}-\mathrm{C}-\mathrm{C}-\mathrm{C}$ or $\mathrm{C}-\mathrm{C}-\mathrm{C}-\mathrm{S})$ was a triple cosine function of the dihedral angle $\varphi$, which has minima corresponding to the trans and gauche conformations. ${ }^{43}$ The nonbonded interatomic interactions were taken to be the Lennard-Jones (LJ) potentials,

$$
V_{\mathrm{LJ}}(r)=4 \varepsilon\left[\left(\frac{\sigma}{r}\right)^{12}-\left(\frac{\sigma}{r}\right)^{6}\right],
$$

where $r$ is the inter-atomic distance; $\varepsilon$ and $\sigma$ are the energy and length parameters, respectively. ${ }^{36}$ The gold-sulfur interaction was taken to be the Morse potential, ${ }^{\mathbf{4 4}}$

$$
V_{\mathrm{Au}-\mathrm{S}}(r)=D \mathrm{e}^{-\alpha(r-b)}\left[\mathrm{e}^{-\alpha(r-b)}-2\right]
$$

where $D$ and $b$ are the well depth and distance at the minimum of the potential energy, respectively. This study used the same LJ and Morse parameters applied in previous simulations. . $3,26,27,45,46$

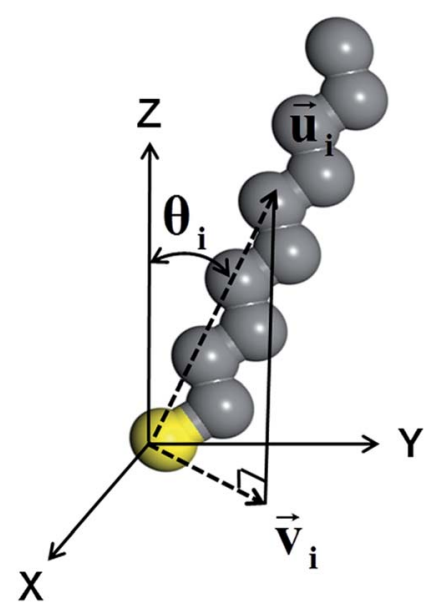

Fig. 1 Orientation of a 1-decanethiol (DT) molecule with all-trans conformations. The tilt direction vector $\vec{u}_{i}$ for the $i^{\text {th }}$ molecule was calculated by selecting $C_{2}, C_{4}$, and $C_{6}$ atoms. $\vec{u}_{i}$ was defined as the average of the direction vectors from the sulfur atom (in yellow) to these selected carbon atoms. $\vec{v}_{i}$ was defined as the surface (XY plane) projection of $\vec{u}_{i}$. The tilt angle, $\theta_{i}$, is the polar angle of $\vec{u}_{i}$ measured from the surface normal ( $Z$ direction).
The MD simulations were run at a constant temperature and volume using the Berendsen thermostat. ${ }^{47}$ The MD trajectories were propagated using the velocity Verlet algorithm with a time step of 1.0 fs. The nonbonded interactions were cut off at a distance of $9.0 \AA$. A parallelepiped simulation box with lattice vectors of $(144.85 \AA$, 83.63 $\AA, 0),(0,167.26 \AA, 0)$ and $(0,0,100.0$ A).

Periodic boundary conditions were applied in the direction parallel to the surface (in $X$ and $Y$ direction). The gold surface, which was comprised of two layers containing 6728 atoms, was fixed in the simulation. A monolayer fully covered with 1159 DT molecules was equilibrated by running a simulation at $300 \mathrm{~K}$ for 3 ns. Circular or linear patterns were next carved out from the relaxed DT monolayer. These engraved monolayers were evolved in time for 40-140 ns. The DLPOLY package ${ }^{48}$ was used to implement the MD methods described above.

The present DT molecule is similar to the thiol molecule previously simulated, ${ }^{23,26,27,45,46}$ differing only in its length. We therefore employed the same force field used in our previous work. The tilt angles of the present molecules in a fully covered monolayer were $22.8^{\circ}$ on average, agreeing with that found in a simulation using a different force field, $23.6^{\circ},{ }^{49}$ and with the experimental values, $20-30^{\circ} .^{50}$ Moreover, the present force field reproduced the packing structures of sulfur atoms of the alkanethiol monolayers measured in experiments. ${ }^{27} \mathrm{~A}$ more sophisticated force field such as the ab initio MD simulation or the reactive force field might give additional features such as the formation of a gold vacancy island ${ }^{51}$ and a dimerization of thiol molecules bridged by a gold atom. ${ }^{52}$ The present force field however captures the essential features of the overall structures of thiol monolayers, as verified in the previous studies.

\section{Results and discussion}

The time development of the circular engravings with diameters of $2.8,3.0,3.2,3.4$, and $3.6 \mathrm{~nm}$ on the DT monolayer was observed. Fig. 2 presents simulation snapshots and tilt direction vectors, $\vec{v}_{i} \mathrm{~s}$, of the circular holes with diameters of 3.0 (a and b) and $3.2 \mathrm{~nm}$ (c and d). In each panel, the MD snapshot and $\vec{v}_{i}$ s are drawn in the top and bottom, respectively. The initial
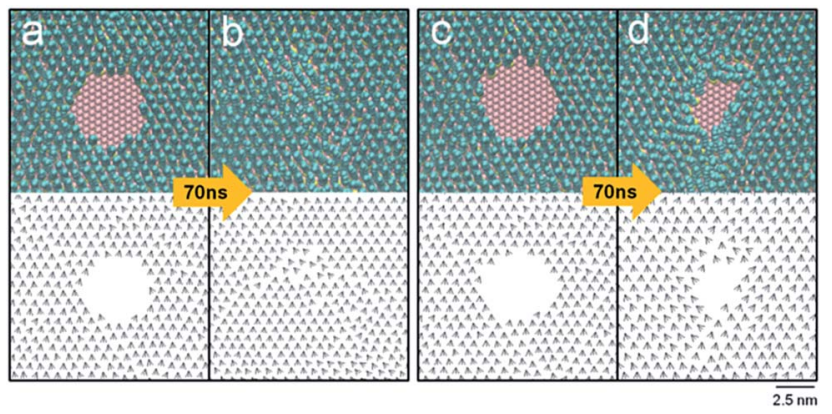

Fig. 2 Time evolution of circular holes engraved on a DT monolayer. In each panel, the simulation snapshot (top) and projected tilt direction vectors $\vec{v}_{i}$ (bottom) are shown for times 0 and 70 ns. Drawn are the holes with initial diameters of 3.0 ( $a$ and b) and 3.2 ( $c$ and d) nm. Only a small portion of the system near the hole is shown for visual clarity. 
$3.0 \mathrm{~nm}$-wide hole disappeared within $60 \mathrm{~ns}$, giving rise to a fully covered monolayer that was less dense than the bulk monolayer. Note that the tilt direction vectors near the region, where the original hole existed, are disordered, as opposed to the almost perfect alignment of the tilt direction vectors in the initial monolayer. The energy of the monolayer increased slightly by $0.1 \mathrm{kcal} \mathrm{mol}^{-1}$ (with a standard deviation of $0.05 \mathrm{kcal}$ $\mathrm{mol}^{-1}$ with time) as the hole vanished spontaneously (Fig. 2a and b). The change in the Helmholtz free energy $A$ is given, in terms of the energy $U$ and entropy $S$ of the system, by $\Delta A=\Delta U-$ $T \Delta S$. As the spontaneous merging or collapse of linear trenches $(\Delta A<0)$ is accompanied by the increase in the energy $(\Delta U>0)$, the entropy must increase to make the overall change spontaneous. Hence, this vanishing is entropy driven, which is consistent with the disorder in the tilt direction vectors shown in Fig. $2 b$.

By contrast, the larger hole (of $3.2 \mathrm{~nm}$ in diameter) survived after 70 ns (Fig. 2c and d). Note, although the individual snapshot shown in Fig. $2 d$ is noncircular, the shape of the pattern averaged over many snapshots is circular. The structural parameters of the hole were calculated by averaging over 500 snapshots taken from 60 to $70 \mathrm{~ns}$. The average tilt angle, $\theta$, was $24^{\circ}$, agreeing with the typical experimental values of the bulk monolayers $\left(20-30^{\circ}\right) .^{50}$ The percentage of trans conformations was $97 \%$ on average. This is slightly lower than the bulk value of $99 \%$, which was previously calculated for ODT. ${ }^{53}$ The order parameter of the molecular orientation, $O_{\mathrm{u}}$, was 0.95 , which is close to perfect ordering (1.0). These average structural parameters are dominated by the majority of molecules far away from the region, where the initial circular hole existed. In the region with radial distances less than $2.6 \mathrm{~nm}$ from the center of the initial hole, the average tilt angle was $25.1^{\circ}$, the percentage of trans conformations was $92 \%$, and $O_{\mathrm{u}}$ was 0.85 , indicating the locally disordered structure. Even larger holes with diameters of 3.4 and $3.6 \mathrm{~nm}$ were stable with respect to time. Therefore, a stable hole should be at least $3.2 \mathrm{~nm}$ in diameter. This should set the theoretical limit to the smallest circular engraving on a DT monolayer. Interestingly, this minimum size is almost twice of that of a positive pattern of an ODT monolayer, $1.9 \mathrm{~nm}^{26}$ The present simulation considered DT, but a shorter or a longer thiol molecule, whose number of carbon atoms ranges from 6 to 24, is also utilized widely. In the case of a bulk monolayer of alkanethiol, an increased length of a thiol molecule, owing to an increased van der Waals intermolecular interaction, gives an enhanced order, packing, and stability of the resulting monolayer. ${ }^{\mathbf{1 0}, 54}$ The tilt angles of molecules were found to increase slightly with increasing the chain length of alkanethiol. ${ }^{49,55}$ For a nanoscale monolayer of alkanethiol, the percentage of trans conformations increased with increasing the chain length. ${ }^{56}$ For the present engraved patterns, the minimum size of such a pattern should decrease with increasing the chain length of alkanethiol, owing to the increased intermolecular interaction. Therefore, the minimum size for ODT will be smaller than the present value found for DT. An exact quantification of this decrease in the minimum size of a nanoscale engraving calls for a separate investigation and is beyond the scope of the present work.
The time-dependent areas of circular holes were investigated (Fig. 3). The area of a hole was calculated by averaging over the radial distances of sulfur atoms located at the periphery of the hole. A sulfur atom was taken to be located at the periphery if the number of neighboring sulfur atoms (within a distance $<6.0$ A) was less than 6 . With increasing time, a circular hole with a diameter $\geq 3.2 \mathrm{~nm}$ decreased gradually and converged to a constant with fluctuations arising from the thermal motion of molecules. For example, the hole with a diameter of $3.2 \mathrm{~nm}$ converged to $453 \AA^{2}$ after $60 \mathrm{~ns}$ (the converged value was calculated by averaging over 500 snapshots taken from 60 to 70 $\mathrm{ns})$. On the other hand, the two smallest holes with diameters of 2.8 and $3.0 \mathrm{~nm}$, respectively, reduced their areas abruptly at 40 and $60 \mathrm{~ns}$ and then decayed gradually to zero.

This study also investigated how close two circular holes can be without merging, which is relevant to the best spatial resolution of engraved patterns. Fig. 4 shows two contrasting cases: two holes with diameters of $3.2 \mathrm{~nm}$ coalesce (a to c), and remain separated ( $b$ to d) depending on their separation. The holes, initially $1.8 \mathrm{~nm}$ apart (periphery-to-periphery distance), merged into a single hole after 120 ns. In contrast, the same holes initially $2.0 \mathrm{~nm}$ apart remained separate even after $140 \mathrm{~ns}$.

The merging of two circular holes was monitored by checking the tilt direction vectors, $\vec{v}_{i} \mathrm{~s}$, as shown in Fig. 5. With increasing time, the concave bridge between the holes (shown in yellow color) was distorted (Fig. $5 \mathrm{~b}$ and $\mathrm{c}$ ), became slender (Fig. 5d), and disappeared, eventually producing a single trench at $100 \mathrm{~ns}$ (Fig. 5e). Note that the molecules constituting the concave bridge are dispersed, and are mostly relocated to the periphery of the single merged trench (Fig. 5e and f). In this merging process (Fig. 5a to e), the tilt direction vectors became disordered near the periphery of the final trench. This indicates the entropy-driven nature of merging, which is also shown in the $0.18 \mathrm{kcal} \mathrm{mol}^{-1}$ increase in the system energy. With further evolution of time, the noncircular trench became an almost circular hole with a diameter of $4.4 \mathrm{~nm}$ at $120 \mathrm{~ns}$.

Linear engravings on the DT monolayer, which are also common motifs of engraved patterns, were next examined. A

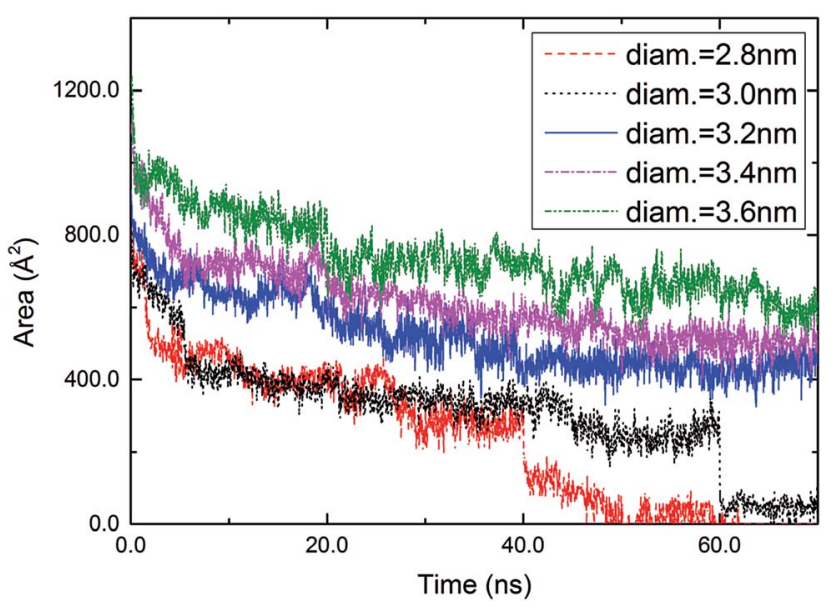

Fig. 3 Time-dependent areas of circular holes with diameters of 2.8, 3.0, 3.2, 3.4, and $3.6 \mathrm{~nm}$ engraved in a DT monolayer. 

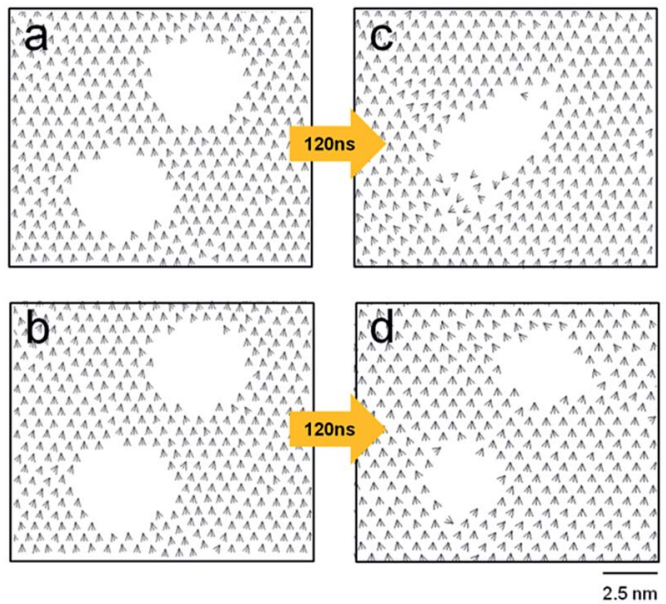

Fig. 4 Coalescence of circular holes at short distances. In the top (a and $c$ ) and bottom ( $b$ and d) panels, two holes with a diameter of $3.2 \mathrm{~nm}$ were initially separated by 1.8 and $2.0 \mathrm{~nm}$ (from the periphery to periphery), respectively. Drawn are the projected tilt vectors, $\vec{v}_{i} s$ taken at 0 ns ( $a$ and $b)$ and 120 ns (c and d).
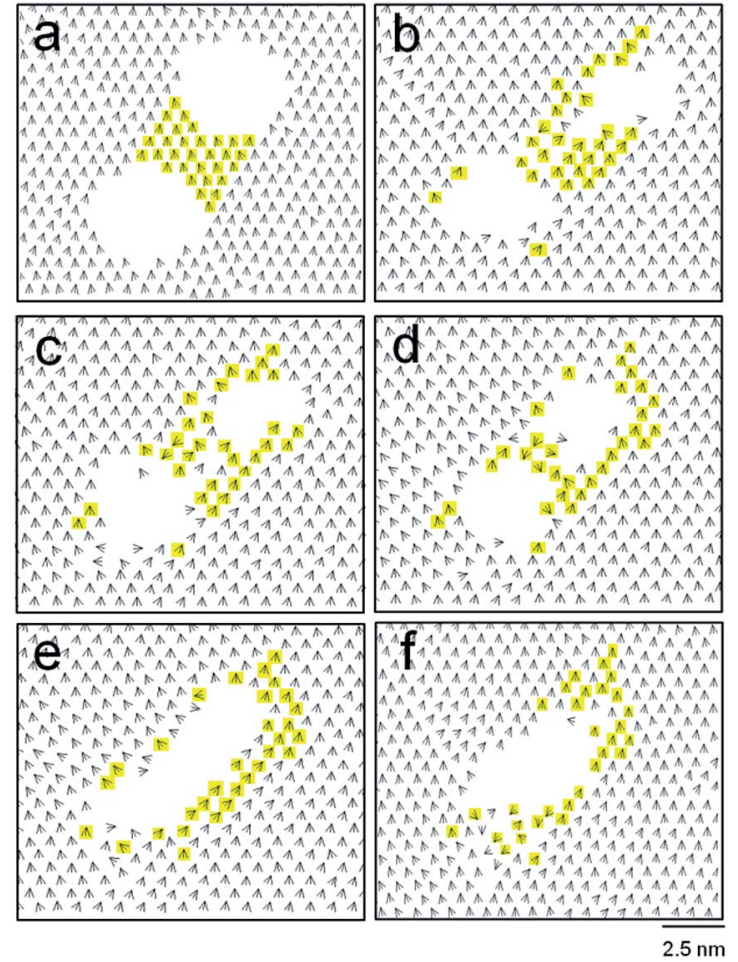

Fig. 5 Merging process of two holes (with diameters of $3.2 \mathrm{~nm}$ ) that are initially $1.8 \mathrm{~nm}$ apart. The tilt direction vector $\vec{v}_{i} \mathrm{~s}$ are shown for 0 (a), 60 (b), 80 (c), 90 (d), 100 (e), and 120 (f) ns. The molecules initially bridging the two holes appear in yellow color.

linear trench was taken to be parallel to one of the unit cell vectors of the gold (111) surface. This way, infinitely long trenches can be simulated by applying the periodic boundary conditions. Fig. 6 shows the time variance of the tilt direction vectors for trenches with different widths, 1.5 (a), 1.8 (b), 2.1 (c), and 2.4 (d) $\mathrm{nm}$. With time, the narrowest trench was partially

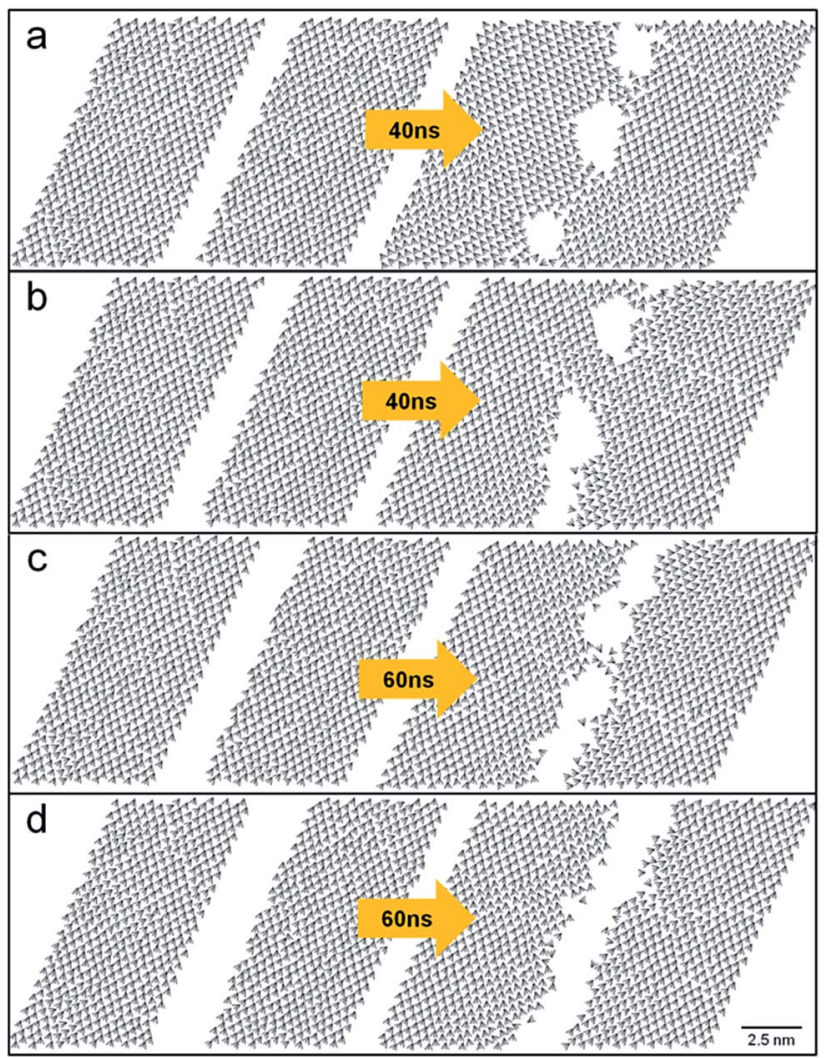

Fig. 6 Time evolution of linear trenches on the DT monolayer with different widths, 1.5 (a), 1.8 (b), 2.1 (c), and $2.4 \mathrm{~nm}$ (d). Drawn are the tilt direction vectors of $\vec{v}_{i}$.

filled with molecules, forming three holes after $40 \mathrm{~ns}$. The 1.8 nm-wide trench also collapsed after $40 \mathrm{~ns}$ (Fig. 6b). For the 2.1 nm-wide trench, only several DT molecules penetrated the trench, bridging the opposite sides of the trench after $60 \mathrm{~ns}$. These collapses of the linear trenches were entropy-driven because the system energies increased by $0.21-0.33 \mathrm{kcal}$ $\mathrm{mol}^{-1}$. With a width of $2.4 \mathrm{~nm}$ or larger, a linear trench was robust to thermal fluctuations after $60 \mathrm{~ns}$ (Fig. 6d). This sets the minimum width required for the stable lines engraved on a DT monolayer. This value $(2.4 \mathrm{~nm})$ is actually larger than the minimum width found for the positive (embossed) lines of an ODT monolayer, $1.7 \mathrm{~nm}^{23}$ The structural parameters of this stable trench $(2.4 \mathrm{~nm}$ in width) were slightly less ordered than those of the bulk monolayer. The average tilt angle, $\theta$, percentage of trans conformations, and $O_{\mathrm{u}}$ were $24.78^{\circ}, 95.74 \%$, and 0.90 , respectively. Near the peripheries of the linear trenches, however, DT molecules are more disordered than the majority of molecules far away from the trenches.

This study investigated two parallel linear trenches by varying their separation to estimate the ultimate spatial resolution of these patterns. Fig. 7 presents the cases where two linear trenches are initially 1.4, 2.0, 2.3, and $2.6 \mathrm{~nm}$ apart. After $40 \mathrm{~ns}$, two parallel trenches merged if their initial separation was less than $2.6 \mathrm{~nm}$. This merging of nearby trenches (Fig. 7ac) were again entropy-driven because the energy of the system increased by $0.19-0.32 \mathrm{kcal} \mathrm{mol}^{-1}$. The monolayer with two 


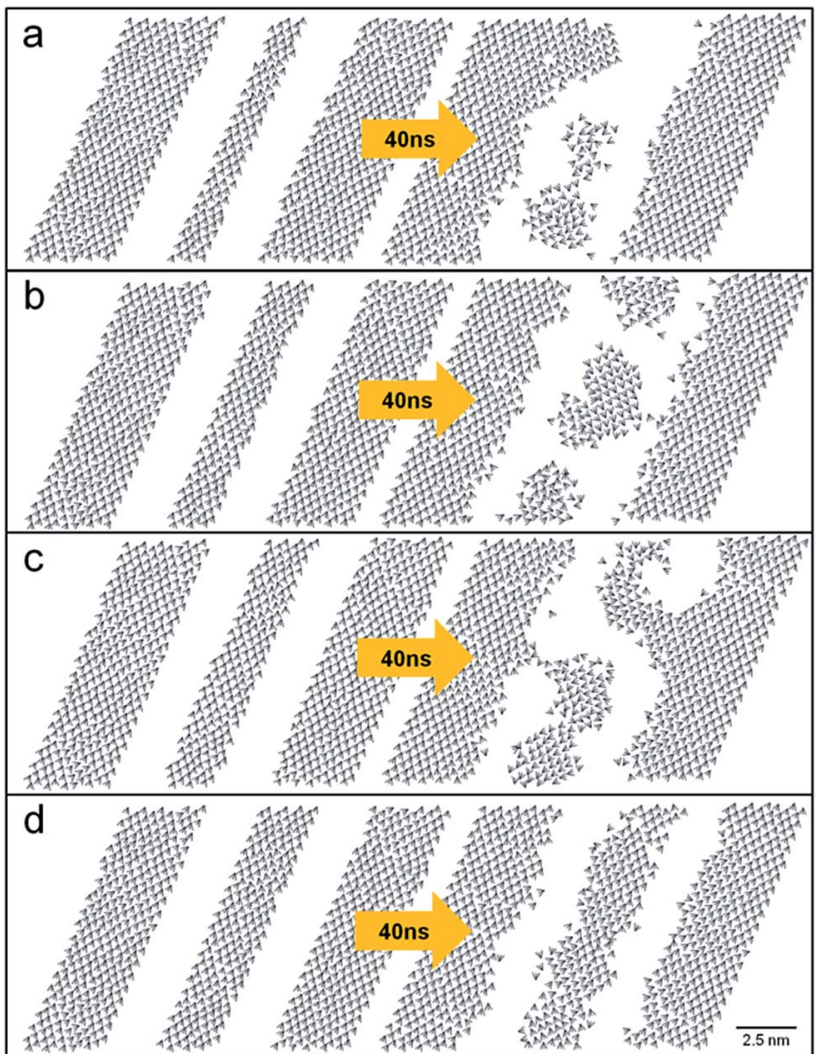

Fig. 7 Two parallel linear engravings on the DT monolayer with various separations between them. Two $2.4 \mathrm{~nm}$ wide trenches were initially separated by 1.4 (a), 2.0 (b), 2.3 (c), and 2.6 (d) nm. Drawn are the tilt direction vectors of $\vec{v}_{i}$ s taken at 0 (left) and 40 (right) ns.

trenches $2.6 \mathrm{~nm}$ apart showed robust structural parameters: the average tilt angle, $\theta$, the percentage of trans conformations, and $O_{\mathrm{u}}$ were $24.70^{\circ}, 93 \%$, and 0.87 , respectively. Therefore, the best spatial resolution of the lines engraved on the DT monolayer was $2.6 \mathrm{~nm}$.

Note the collapse of an engraved pattern is a catastrophic event involving a sudden drop in its area as shown in Fig. 3. We checked whether such a catastrophic event occurs later by further running simulation for extra $30 \mathrm{~ns}$, but the circular hole with a diameter of $3.2 \mathrm{~nm}$ in Fig. 2 remained robust. We also confirmed the two holes in Fig. 4 remain separate from each other by running simulation for extra 140 ns. Nevertheless, due to the inherent limitation of a finite MD simulation time, we cannot ensure that these patterns will survive for a much longer timescale such as $>1 \mathrm{~ms}$. For this purpose, an efficient sampling method such as the Monte Carlo method m $^{57,58}$ or accelerated MD simulation $^{59}$ might be needed.

\section{Conclusions}

MD simulations were performed to investigate the nanometersized patterns engraved on the DT monolayer on a gold surface. This study examined circular and linear engravings, which are common motifs produced experimentally. The ultimate limits for the size and spatial resolution for these negative patterns were estimated by examining circular and linear engravings with various sizes and separations. A stable circular hole and linear trench need to have minimum widths of 3.2 and $2.4 \mathrm{~nm}$, respectively; the neighboring holes and linear trenches merge if their distances are less than 2.0 and $2.6 \mathrm{~nm}$, respectively. These results place theoretical limits on the everdecreasing sizes of nanoscale engravings on alkanethiol monolayers.

\section{Conflict of interest}

There are no conflicts to declare.

\section{Acknowledgements}

This study was supported by the National Research Foundation of Korea (NRF) grant funded by the Korean government (NRF2015R1A2A2A01004208, NRF-2014R1A4A1001690, and NRF2016H1D3A1936765).

\section{Notes and references}

1 A. Kumar, N. L. Abbott, H. A. Biebuyck, E. Kim and G. M. Whitesides, Acc. Chem. Res., 1995, 28, 219-226.

2 L. Bumm, J. Arnold, M. Cygan, T. Dunbar, T. Burgin, L. Jones, D. Allara, J. M. Tour and P. Weiss, Science, 1996, 271, 17051707.

3 D. C. Kim and D. J. Kang, Sensors, 2008, 8, 6605-6641.

4 Z. Zhao, I. A. Banerjee and H. Matsui, J. Am. Chem. Soc., 2005, 127, 8930-8931.

5 E. Ul-Haq, S. Patole, M. Moxey, E. Amstad, C. Vasilev, C. N. Hunter, G. J. Leggett, N. D. Spencer and N. H. Williams, ACS Nano, 2013, 7, 7610-7618.

6 K. Wadu-Mesthrige, S. Xu, N. A. Amro and G.-Y. Liu, Langmuir, 1999, 15, 8580-8583.

7 M. N. Szöri, D. J. Tobias and M. Roeselová, J. Phys. Chem. B, 2009, 113, 4161-4169.

8 V. Vasumathi and M. N. D. Cordeiro, Chem. Phys. Lett., 2014, 600, 79-86.

9 C. Singh, P. K. Ghorai, M. A. Horsch, A. M. Jackson, R. G. Larson, F. Stellacci and S. C. Glotzer, Phys. Rev. Lett., 2007, 99, 226106.

10 J. Zhao and K. Uosaki, Nano Lett., 2002, 2, 137-140.

11 J. M. Tour, Acc. Chem. Res., 2000, 33, 791-804.

12 Y. Xia and G. M. Whitesides, J. Am. Chem. Soc., 1995, 117, 3274-3275.

13 R. J. Jackman, J. L. Wilbur and G. M. Whitesides, Science, 1995, 269, 664-666.

14 A. Kumar, H. A. Biebuyck, N. L. Abbott and G. M. Whitesides, J. Am. Chem. Soc., 1992, 114, 9188-9189.

15 C. A. Mirkin, ACS Nano, 2007, 1, 79-83.

16 R. D. Piner, J. Zhu, F. Xu, S. Hong and C. A. Mirkin, Science, 1999, 283, 661-663.

17 S. Xu, S. Miller, P. E. Laibinis and G.-Y. Liu, Langmuir, 1999, 15, 7244-7251.

18 X.-D. Xiao, G.-Y. Liu, D. H. Charych and M. Salmeron, Langmuir, 1995, 11, 1600-1604. 
19 G.-Y. Liu, S. Xu and Y. Qian, Acc. Chem. Res., 2000, 33, 457466.

20 J. C. Love, L. A. Estroff, J. K. Kriebel, R. G. Nuzzo and G. M. Whitesides, Chem. Rev., 2005, 105, 1103-1170.

21 S. Hong and C. A. Mirkin, Science, 2000, 288, 1808-1811.

22 P. Campiglio, M. Campione and A. Sassella, J. Phys. Chem. C, 2009, 113, 8329-8335.

23 J. K. Saha, H. Kim and J. Jang, J. Phys. Chem. C, 2012, 116, 25928-25933.

24 S. Xu and G.-y. Liu, Langmuir, 1997, 13, 127-129.

25 B. Rai, P. Sathish, C. P. Malhotra, A. Pradip and K. Ayappa, Langmuir, 2004, 20, 3138-3144.

26 J. K. Saha, Y. Ahn, H. Kim, G. C. Schatz and J. Jang, J. Phys. Chem. C, 2011, 115, 13193-13199.

27 Y. Ahn, J. K. Saha, G. C. Schatz and J. Jang, J. Phys. Chem. C, 2011, 115, 10668-10674.

28 H. H. Cao, N. Nakatsuka, A. C. Serino, W.-S. Liao, S. Cheunkar, H. Yang, P. S. Weiss and A. M. Andrews, ACS Nano, 2015, 9, 11439-11454.

29 E. A. Josephs and T. Ye, J. Am. Chem. Soc., 2010, 132, 1023610238.

30 C. Xu, S. Peng, G. Qiao and X. Zhang, Langmuir, 2016, 32, 11197-11202.

31 S.-S. Li, L.-P. Xu, L.-J. Wan, S.-T. Wang and L. Jiang, J. Phys. Chem. B, 2006, 110, 1794-1799.

32 C. Chu, J.-S. Na and G. N. Parsons, J. Am. Chem. Soc., 2007, 129, 2287-2296.

33 O. Lyandres, N. C. Shah, C. R. Yonzon, J. T. Walsh, M. R. Glucksberg and R. P. Van Duyne, Anal. Chem., 2005, 77, 6134-6139.

34 L. Y. S. Lee, T. C. Sutherland, S. Rucareanu and R. B. Lennox, Langmuir, 2006, 22, 4438-4444.

35 S. Chen, L.-P. Xu, S. Pradhan and W. Chen, Solid State Commun., 2007, 144, 124-127.

36 J. Hautman and M. L. Klein, J. Chem. Phys., 1989, 91, 49945001.

37 P. K. Ghorai and S. C. Glotzer, J. Phys. Chem. C, 2007, 111, 15857-15862.

38 W. Mar and M. L. Klein, Langmuir, 1994, 10, 188-196.

39 J. P. Bareman and M. L. Klein, J. Phys. Chem., 1990, 94, 52025205.
40 R. Bhatia and B. J. Garrison, Langmuir, 1997, 13, 4038-4043. 41 S. Fujiwara and T. Sato, J. Chem. Phys., 1999, 110, 9757-9764. 42 W. L. Jorgensen, D. S. Maxwell and J. Tirado-Rives, J. Am. Chem. Soc., 1996, 118, 11225-11236.

43 W. L. Jorgensen, J. D. Madura and C. J. Swenson, J. Am. Chem. Soc., 1984, 106, 6638-6646.

44 X. Zhao, Y. Leng and P. T. Cummings, Langmuir, 2006, 22, 4116-4124.

45 Z. Zhang, H. Kim, J. Noh, Y. Ahn, J. Y. Son and J. Jang, Nanoscale, 2016, 8, 1133-1139.

46 Z. Zhang, Y. Ahn, J. Y. Son and J. Jang, J. Phys. Chem. C, 2016, 120, 15509-15513.

47 H. J. Berendsen, J. P. M. Postma, W. F. van Gunsteren, A. DiNola and J. Haak, J. Chem. Phys., 1984, 81, 3684-3690.

48 W. Smith, C. Yong and P. Rodger, Mol. Simul., 2002, 28, 385471.

49 L. Ramin and A. Jabbarzadeh, Langmuir, 2011, 27, 97489759.

50 M. D. Porter, T. B. Bright, D. L. Allara and C. E. Chidsey, J. Am. Chem. Soc., 1987, 109, 3559-3568.

51 G. S. Longo, S. K. Bhattacharya and S. Scandolo, J. Phys. Chem. C, 2012, 116, 14883-14891.

52 V. Vasumathi, J. L. C. Fajin and M. N. D. S. Cordeiro, J. Phys.: Conf. Ser., 2014, 5, 1441011.

53 H. Kim, J. K. Saha, Z. Zhang, J. Jang, M. A. Matin and J. Jang, J. Phys. Chem. C, 2014, 118, 11149-11157.

54 N. Prathima, M. Harini, N. Rai, R. Chandrashekara, K. Ayappa, S. Sampath and S. Biswas, Langmuir, 2005, 21, 2364-2374.

55 Y. Wang, J. Solano Canchaya, W. Dong, M. Alcami, H. Busnengo and F. Martin, J. Phys. Chem. A, 2014, 118, 4138-4146.

56 J. K. Saha, M. Yang and J. Jang, Bull. Korean Chem. Soc., 2013, 34, 3790-3794.

57 E. O. Fetisov and J. I. Siepmann, J. Phys. Chem. B, 2016, 120, 1972-1978.

58 O. Alexiadis, K. C. Daoulas and V. G. Mavrantzas, J. Phys. Chem. B, 2008, 112, 1198-1211.

59 H. Morgner, Langmuir, 1997, 13, 3990-4002. 